\title{
The effect of fatty meal and water on interfering extracardiac activity in myocardial perfusion single-photon emission computed tomography
}

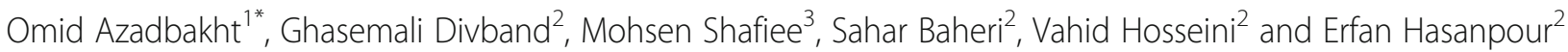

\begin{abstract}
Background: There are several ways to diagnose coronary artery disease. Myocardial perfusion imaging (MPI) is used for the diagnosis of coronary artery disease. One of the practical challenges in scan's interpretation is interfering liver radiotracer uptake with appropriate cardiac visualization. For many years, different approaches were evaluated for conquering this problem. A relatively common method is using fat-containing meals before imaging. Fatty meals may cause some problems for some patients, especially elderly with digestive disorders, as well as an extra cost. We designed a study to compare the efficacy of water and fatty meal as a liver clearing agents to facilitate MPI results interpretation.
\end{abstract}

Result: Totally, 30 patients (consist of 30 stress phases and 30 rest phases), including 18 women and 12 men, were evaluated. The fatty meal was used before 32 scans and cold water in the other 28 scans. Finally, 40 scans had the suitable quality for reporting by nuclear physicians among 60 scans, which included 20 of 28 scans with water and 20 of 32 scans with a fatty meal.

Conclusions: This comparison showed that scans performed with the water group had similar results with the fatty meal group. Water is better than fatty meals because of its ease of use and lower cost. Therefore, the use of water can be a good alternative for routine use in NM centers.

Keywords: MPI, Water, Fatty Meal, Coronary artery disease, NM centers

\section{Background}

Coronary artery disease is a common disease in the world. Different methods such as exercise testing, electrocardiogram, single-photon diffusion tomography (SPECT), and cardiac angiography are used to diagnose CAD [1, 2]. Myocardial perfusion imaging (MPI) is widely used as a non-invasive clinical imaging technique for the diagnosis of coronary artery disease [3, 4, 5]. MPI in nuclear medicine consists of two steps: first is rest and the

\footnotetext{
* Correspondence: omidazadbakht68@gmail.com

1 Department Of Radiology Technology, Behbahan Faculty of Medical

Sciences, Behbahan, Iran

Full list of author information is available at the end of the article
}

other is stress. Stress stage is done in two ways (drug stress or exercise).

In this phase, blood flow should increase. If the tide is reduced or limited, the vessels will be involved. The results are usually compared to the rest phase [6]. Two most commonly used radiopharmaceuticals in heart scans are Tc-99m sestamibi and Tc-99m tetrofosmin. For sestamibi, tetrofosmin, and thallium, uptake in the myocardium is proportional to blood flow. Both tetrofosmin and sestamibi are excited from the body through the hepatobiliary system into the intestine [7-10]. The heart is enclosed among the diaphragm and the intestine and the liver, and it is 
Table 1 Sex

\begin{tabular}{|c|c|c|c|c|c|c|}
\hline Report Quality & & & Frequency & Percent & Valid percent & Cumulative percent \\
\hline \multirow[t]{3}{*}{ Reportable } & Valid & Male & 14 & 35.0 & 35.0 & 35.0 \\
\hline & & Female & 26 & 65.0 & 65.0 & 100.0 \\
\hline & & Total & 40 & 100.0 & 100.0 & \\
\hline \multirow[t]{3}{*}{ Non reportable } & Valid & Male & 10 & 50.0 & 50.0 & 50.0 \\
\hline & & Female & 10 & 50.0 & 50.0 & 100.0 \\
\hline & & Total & 20 & 100.0 & 100.0 & \\
\hline
\end{tabular}

Reportable scan examination in male and female patients

located on the diaphragm above the left edge of the liver and near the intestine. For this reason, the liver, bile duct, and intestine cause excessive radiation. Therefore, radioactivity in the subdiaphragm region can change the results in heart scan studies and make it harder for nuclear medicine studies to achieve the desired goal. Due to the proximity of the liver and the intestine to the bottom of the heart, proper judgment can be severe in CAD cases $[11,12]$.

The emergence of unusual cardiac activity is predictable in the imaging of myocardial perfusion, so to provide high-quality images for comment, it is essential to remove the liver-bile duct and digestive tract $[3,7,13]$.

Some foods and fluids have been used to reduce or eliminate this problem, which they increase radiopharmaceutical hepatobiliary and intestine transition, including drinking milk, eating high-fat foods, drinking milk and water, injections of cholecystokinin, and administration of metoclopramide in myocardial perfusion SPECT. Fatty foods release cholecystokinin (CCK) and increase secretion bile and evacuate the gallbladder whereby increased clearance hepatobiliary and reduced infracardiac activity $[1,11,14,15]$. The main route of excretion of technetium, the liver, and the secondary route is the gastrointestinal tract. Increasing age leads to a decrease in the performance of these two systems. Increasing age leads to a decrease in the ability of the liver system to purify the material. Digestive motions of the gastrointestinal tract become ineffective as they age. As a result, increased intake of fatty acids leads to gastrointestinal complications in the elderly. On the other hand, a decrease in the function of these systems results in a decrease in the clearance of radioactive substances from the body of the elderly. In a study by Hofman et al., the group received the milk compared to the group that received the water, radiation was significantly reduced, but it did not improve the interpretation of the images [16-18]. The study by Boz et al. indicates that eating solid and liquid foods before the resting stage reduced intestinal activity in Tc-99m tetrofosmin myocardial perfusion $[19,20]$. Also, Purbhoo et al. showed that diluted lemon juice and milk reduced significantly infracardiac radiation in myocardial perfusion scan with radiopharmaceuticals Tc-99m sestamibi, which this reduction in the milk group had been more prominent [21]. The purpose of our studies was to determine what steps should be taken before resting the SPECT myocardium so that we can reduce the side effects of the liver, intestine, and stomach interactions.

\section{Methods}

This study was performed on 30 patients (female /male) who were under MPI. We used the 2-day

Table 2 Scan phase

\begin{tabular}{|c|c|c|c|c|c|c|}
\hline \multicolumn{3}{|l|}{ Report quality } & \multirow{2}{*}{$\frac{\text { Frequency }}{22}$} & \multirow{2}{*}{$\frac{\text { Percent }}{55.0}$} & \multirow{2}{*}{$\begin{array}{l}\text { Valid percent } \\
55.0\end{array}$} & \multirow{2}{*}{$\frac{\text { Cumulative percent }}{55.0}$} \\
\hline Reportable & Valid & Rest & & & & \\
\hline & & Stress & 18 & 45.0 & 45.0 & 100.0 \\
\hline & & Total & 40 & 100.0 & 100.0 & \\
\hline \multirow[t]{3}{*}{ Non Reportable } & Valid & Rest & 8 & 40.0 & 40.0 & 40.0 \\
\hline & & Stress & 12 & 60.0 & 60.0 & 100.0 \\
\hline & & Total & 20 & 100.0 & 100.0 & \\
\hline
\end{tabular}


Table 3 Liver clearance agent

\begin{tabular}{|c|c|c|c|c|c|c|}
\hline $\begin{array}{l}\text { Report } \\
\text { quality }\end{array}$ & & & Frequency & Percent & $\begin{array}{l}\text { Valid } \\
\text { percent }\end{array}$ & Cumulative percent \\
\hline \multirow[t]{3}{*}{ Reportable } & Valid & Water & 20 & 50.0 & 50.0 & 50.0 \\
\hline & & Profiterole & 20 & 50.0 & 50.0 & 100.0 \\
\hline & & Total & 40 & 100.0 & 100.0 & \\
\hline \multirow[t]{3}{*}{ Non Reportable } & Valid & Water & 8 & 40.0 & 40.0 & 40.0 \\
\hline & & Profiterole & 12 & 60.0 & 60.0 & 100.0 \\
\hline & & Total & 20 & 100.0 & 100.0 & \\
\hline
\end{tabular}

Checking for scan reports in different types of liver drainage

protocol and with the same injectable dose. At each phase of the patient's scan (rest or stress), one of the two methods of extracardiac uptake discharge was used. According to previous studies, we used two methods for extracardiac uptake discharge. The first method is the use of profiterole with specific compounds (from butter). According to the recommendation of nuclear medicine technology: procedure and quick reference [6,22], the second method is to use cold water before scanning [6]. For the same physiological conditions of the patient and a better comparison of the two methods of extracardiac uptake discharge, we used two methods for extracardiac uptake discharge in each of the two scan phases (rest, stress). The imaging process was carried out with the gamma camera at $60 \mathrm{~min}$ after the Tc-99m sestamibi injection. Finally, a nuclear medicine specialist reported a "reportable" factor for each scan. It should be noted that the nuclear medicine specialist did not know the protocol performed for each scan stage. The results were analyzed using the statistical software (SPSS 22).

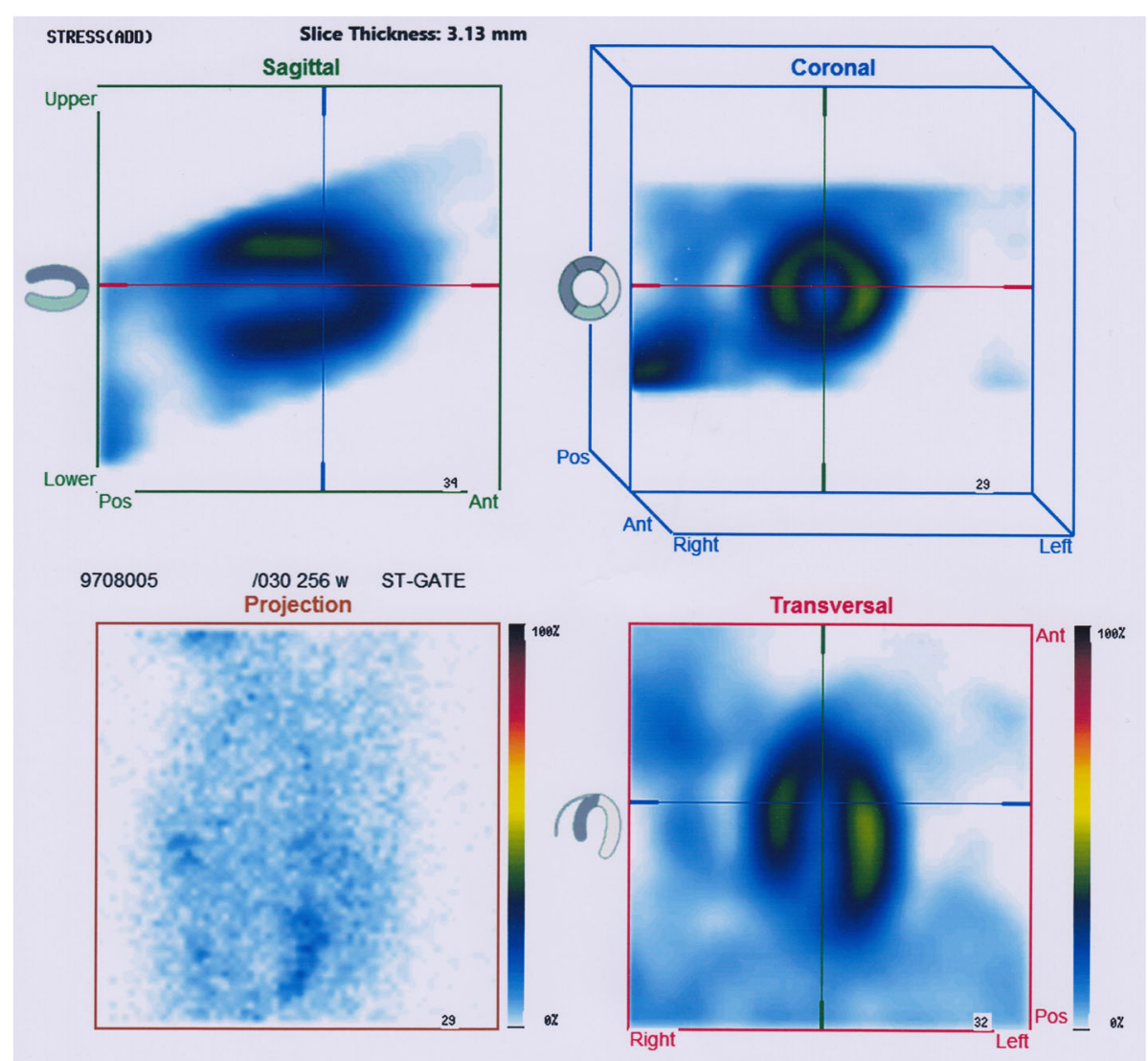

Fig. 1 Fatty meal (Non reportable) 


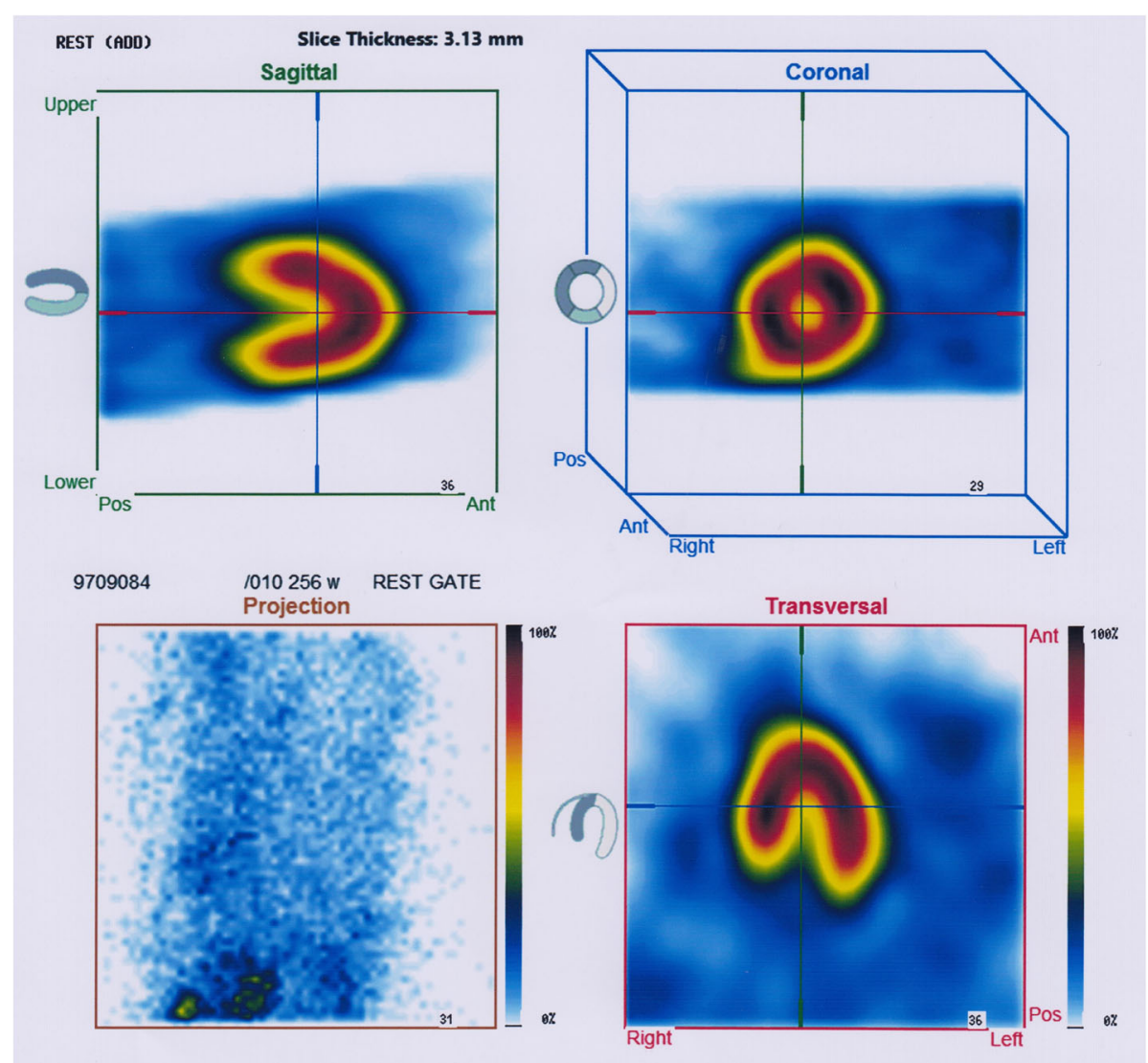

Fig. 2 Fatty meal (Reportable)

We used a single head MIE for doing this research, and the software we used was Sintron 7. Reconstruction of images is done with the ECT program, and the factors used are as follows [23]:

\begin{tabular}{ll}
\hline Filter type & Butter worth \\
\hline Method & FBP \\
Butter worth & $\# 5$ \\
Cutoff frequency & 0.4 \\
\hline
\end{tabular}

\section{Result}

Twelve men and 18 women (30 patients) were examined for this test. Thirty scans were performed in a rest phase and 30 scans in the stress phase. To discharge the liver in a heart test, for each patient, each scan phase used one of two approaches. The two methods are drinking cold water and eating profiterole that was used in 28 scans of water (rest or stress) and 32 scans of the profiterole. We study "reportable factor." From 24 male scans, fourteen men were reportable, and also, 26 women of 36 were reportable (Table 1). Also, we performed 30 scans at rest and 30 scans at stress that 22 of the rest scans and 18 of the stress scans were reportable (Table 2), as well as liver discharge in 28 scans by drinking water and 32 scans by eating the profiterole. Of the 28 scans, 20 scans can be reported, and of the 32 scans, only 20 scans were reported. The description stated in the text indicates that the data were not statistically significant, but those who used water had about $50 \%$ more chance of reporting and using water has better results (Table 3). We show four images of unreported (including fatty meals and water) examples in Figs. 1, 2, 3 and 4. It should be noted that all images of patients were examined by three physicians visually.

\section{Discussion}

In many previous studies, various factors have been investigated by researchers. Things like using the UDCA in the survey of Sood [20] or using different filters in the survey of Shah [17]; however, research on food for better imaging has always been considered. A survey by Gholami et al. found that gender, age, and BMI did not significantly alter spectral imaging quality. On the 


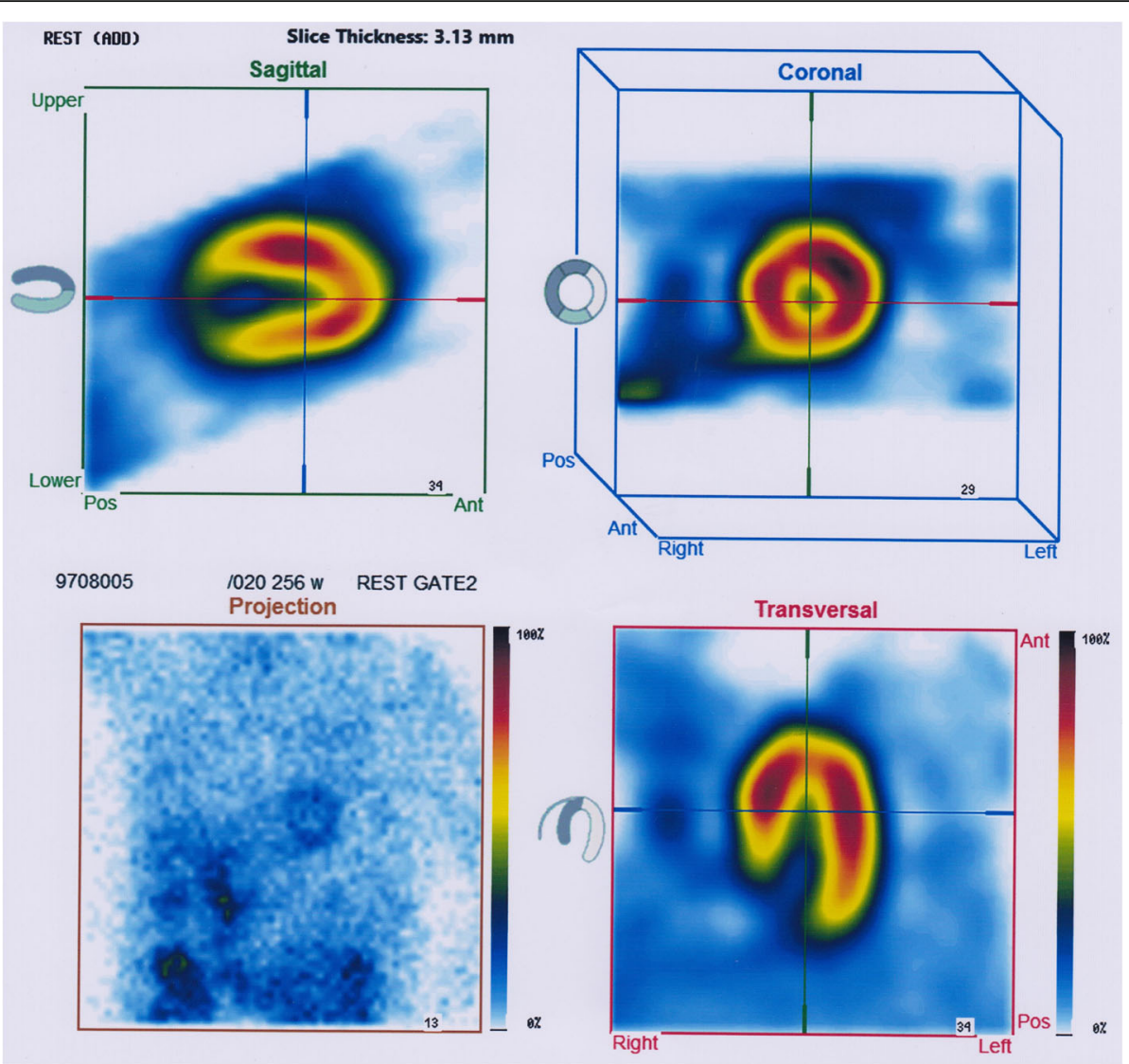

Fig. 3 Water (Non reportable)

other hand, there was a significant difference only in the group consuming milk compared to the control group. This is a confirmation of high-fat milk discharge performance in itself in nuclear medicine scans. Therefore, different substances can be compared to milk [1]. Hara et al. found that soda water increased intestinal activity and increased image quality at the time of imaging [7]. Also, Alice J. Vandongen et al. found that water and milk intake at specific times before scanning facilitated better image acquisition [14]. Boz et al. has been established that the consumption of solid foods and liquids causes gastric filling and changes in bowel movements resulting in better imaging [19]. In a 2017 study, Mohsen Qutbi also showed that consuming 2-4 glasses of water immediately before imaging can eliminate extracardiac activity in cardiac imaging [18]. All of these studies confirm the reason for our use of water as an alternative method of reducing extracardiac activity. In their research, Peace et al. investigated several factors affecting cardiac imaging and ultimately found no significant difference between water and milk intake [8]. We did not differ in the present study. However, in 2005, a survey by Dee Nandurkar and colleagues showed that the effect of drinking milk from consuming water for imaging the heart was more significant in nuclear medicine [16]. A comparison between two common interventions to reduce extracardiac disturbing activity was performed. According to the previous studies, fat meals could prompt radiotracer clearance from the liver. However, fat meals may led to some problems for patients, especially in elderly and in patients with gastrointestinal disorders. So looking for a safe approach with fewer side effects is interested in researchers. We compare cold water versus high-fat meal in this study. Finally, no remarkable difference for diagnostic report of myocardial perfusion imaging was noted among two interventions, but cold water has favorable outcomes with convenience for the patients in comparison to fat congaing meals. Further studies are needed to confirm these results and attempt to consider this approach as a standard intervention in the nuclear cardiology setting. 


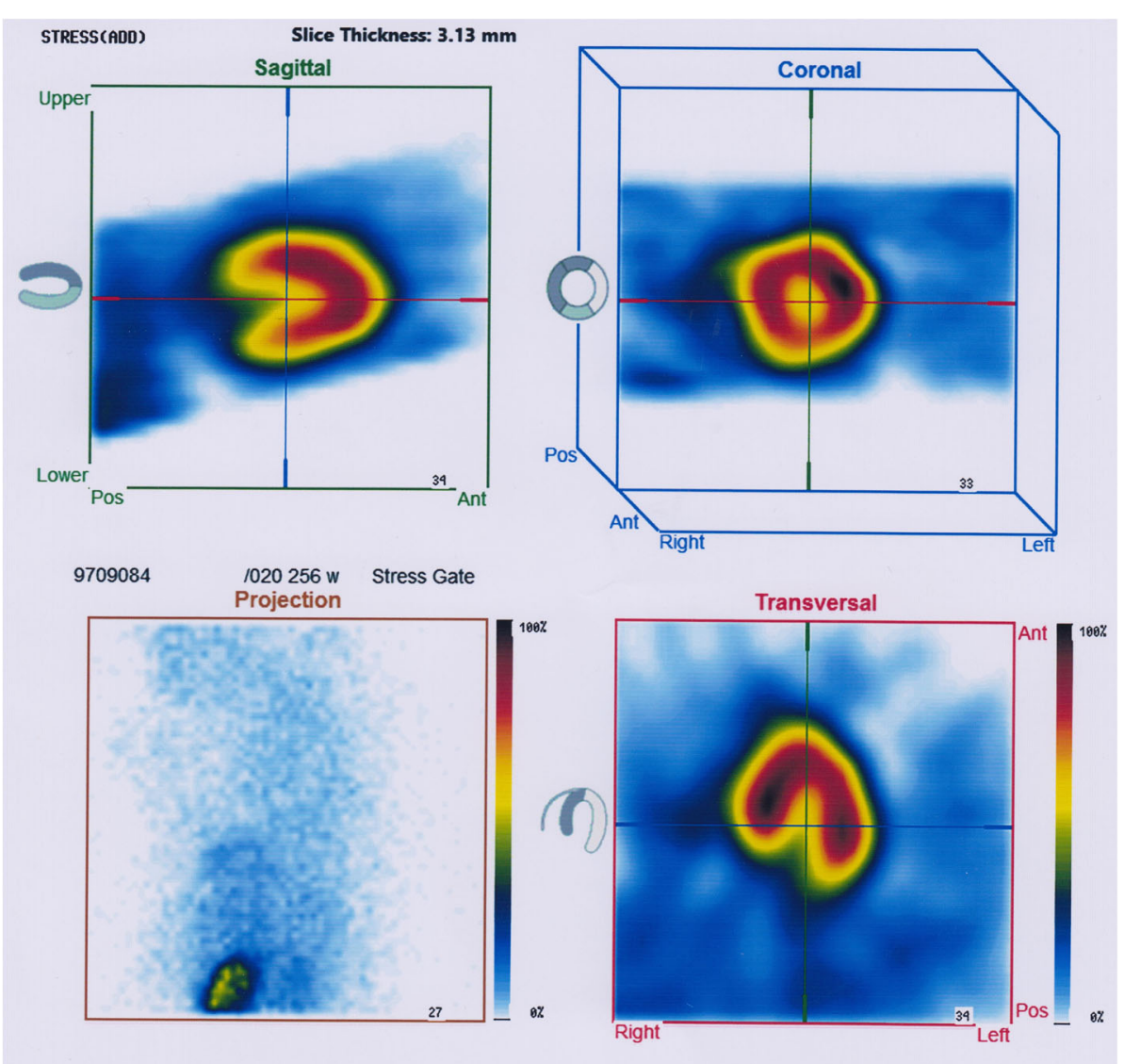

Fig. 4 Water (Reportable)

\section{Conclusions}

The results of this study showed that scans performed with the water groups had equal results with the fatty meal group. Water is better than fatty meals because of its ease of use and lower cost. Therefore, the use of water can be a good alternative for routine use in NM centers. Fatty meals can cause gastrointestinal side effects in patients, especially the elderly, and water is useable for everyone. Therefore, staffs are advised to use water because it has fewer side effects for patients.

\section{Abbreviations}

MPI: Myocardial perfusion imaging: CAD: Coronary Artery Disease; NM: Nuclear medicine; SPECT: Single-photon emission computed tomography; Tc: Technetium; CCK: Cholecystokinin

\section{Acknowledgements}

We would like to extend our thanks to the patients which without their kind cooperation this study would not have been feasible.

\section{Authors' contributions}

$\mathrm{OA}, \mathrm{GHD}, \mathrm{MSH}, \mathrm{SB}, \mathrm{BV}$, and $\mathrm{EH}$ conceived and planned the experiments. OA, GHD, MSH, and SB carried out the experiments. OA, GHD, and MSH planned and carried out the simulations. OA, GHD, MSH, SB, BV, and EH contributed to sample preparation. $\mathrm{OA}, \mathrm{GHD}, \mathrm{MSH}$, and $\mathrm{SB}$ contributed to the interpretation of the results. OA took the lead in writing the manuscript. All authors provided critical feedback and helped shape the research, analysis, and manuscript. All authors read and approved the final manuscript.

\section{Funding}

No funding was obtained for this study.

\section{Availability of data and materials}

The datasets used and/or analyzed during the current study are available from the corresponding author on reasonable request.

\section{Ethics approval and consent to participate}

This study was reviewed by the Ethics Committee of Behbahan School of Medical Sciences. In this study, a physician's instruction was used to reduce the gastrointestinal complications of milk consumption in patients, especially the elderly.

In fact, water has been a facilitator in the imaging of radioactive material. Therefore, the Ethics Committee of Behbahan Paramedical School stated that this study does not require ethical approval.

Informed consent was obtained from patient information.

The results of this study were provided to the participants.

\section{Consent for publication}

All patients included in this research gave written informed consent to publish the data contained within this study. In this study, informed consent was obtained from all participants. The patient was illiterate or unconscious when consent for publication was requested and written informed consent for the publication of this data was given by their parent or legal guardian.

\section{Competing interests}

We declare that we have no significant competing financial, professional, or personal interests that might have influenced the performance or presentation of the work described in this manuscript.

We have described us potential competing financial, professional, and/or personal interests in the space. 


\section{Author details}

'Department Of Radiology Technology, Behbahan Faculty of Medical Sciences, Behbahan, Iran. 'Behbahan Faculty of Medical Sciences, Behbahan, Iran. ${ }^{3}$ Department of Nursing, Abadan Faculty of Medical Sciences, Abadan, Iran

Received: 11 December 2019 Accepted: 17 March 2020

Published online: 06 May 2020

\section{References}

1. Mousavie Anijdan S, Gholami A (2018) An evaluation of the effectiveness of water, milk and natural lemon juice in hepatic biliary secretion of 99mtcmibi radiopharmaceutical in myocardial perfusion imaging. J Babol Univ Med Sci 20(1):12-17

2. Iskandrian AE (2019) Left Ventricular perfusion and function at the crossroads. J Nucl Cardiol:1-2

3. Johansen A, Lomsky M, Gerke O, Edenbrandt L, Johansson L, Hansen G et al (2013) When is reacquisition necessary due to high extra-cardiac uptake in myocardial perfusion scintigraphy? EJNMMI Res 3(1):20

4. Tadehara F, Yamamoto H, Tsujiyama S, Hinoi T, Matsuo S, Matsumoto N et al (2008) Feasibility of a rapid protocol of 1-day single-isotope rest/ adenosine stress Tc-99 m sestamibi ECG-gated myocardial perfusion imaging. J Nucl Cardiol 15(1):35-41

5. Singh A (2017) A retrospective analysis of the utility of myocardial perfusion imaging using single photon emission computed tomography (SPECT) for differentiating ischaemic from non-ischaemic left ventricular dysfunction

6. Shackett $P$ (2019) Nuclear medicine technology: procedures and quick reference. Lippincott Williams \& Wilkins

7. Hara M, Monzen H, Futai R, Inagaki K, Shimoyama H, Morikawa M et al (2008) Reduction of infracardiac intestinal activity by a small amount of soda water in technetium-99 m tetrofosmin myocardial perfusion scintigraphy with adenosine stress. J Nucl Cardiol 15(2):241

8. Peace RA, Lloyd JJ (2005) The effect of imaging time, radiopharmaceutical, full fat milk and water on interfering extra-cardiac activity in myocardial perfusion single photon emission computed tomography. Nucl Med Commun 26(1):17-24

9. Sridhara BS, Braat S, Rigo P, Itti R, Cload P, Lahiri A et al (1993) Comparison of myocardial perfusion imaging with technetium- $99 \mathrm{~m}$ tetrofosmin versus thallium-201 in coronary artery disease. Am J Cardiol 72(14):1015-1019

10. Farzanefar S, Mirzabeigi A, Abbasi M (2016) The incidental lung lesion: effect of cardiac stress on the MIBI uptake in the lung mass. Int J Radiat Res 14(1): 77-79

11. Yaghoobi N, Mardanshahi A, Rastgou F, Malek H, Firouzabady S-H, Rajabi AB et al (2012) Impact of metoclopramide on image quality in myocardial perfusion imaging. Nucl Med Commun 33(10):1053-1057

12. Heller EN, DeMan P, Liu Y-H, Dione DP, Zubal IG, Wackers FJ et al (1997) Extracardiac activity complicates quantitative cardiac SPECT imaging using a simultaneous transmission-emission approach. J Nucl Med 38(12):1882-1890

13. Haghighatafshar M, Sarfaraz A, Banani A, Farhoudi F, Etemadi Z (2019) The effect of pomegranate juice, lemon juice and secanjabin in reducing infracardiac activity of $99 \mathrm{mTc}-\mathrm{MIBI}$ during myocardial perfusion imaging in comparison with fatty food. Iran J Nucl Med 27(2):113-117

14. Van Dongen AJ, Van Rijk PP (2000) Minimizing liver, bowel, and gastric activity in myocardial perfusion SPECT. J Nucl Med 41(8):1315-1317

15. Darkhor P, Mahmoudian B, Gharepapagh E, Zakavi SR, Pirayesh IJ (2018) A study on differentiation of extra-cardiac activity by slit slat collimation in single photon emission computed tomography. Iran J Nucl Med 26(1):22-29

16. Hofman M, McKay J, Nandurkar D (2006) Efficacy of milk versus water to reduce interfering infra-cardiac activity in 99mTc-sestamibi myocardial perfusion scintigraphy. Nucl Med Commun 27(11):837-842

17. Shah A, DePuey E, Friedman M (2016) Intense moving intestinal activity as a source of artifact on myocardial perfusion SPECT study. J Nucl Cardiol 23(1): $161-167$

18. Qutbi M (2018) A simple practical technique to alleviate the problem of interfering subdiaphragmatic intestinal activity on myocardial perfusion imaging. J Nucl Cardiol 25(2):693-697

19. Boz A, Gungor F, Karayalçın B, Yıldız A (2003) The effects of solid food in prevention of intestinal activity in Tc-99 $\mathrm{m}$ tetrofosmin myocardial perfusion scintigraphy. J Nucl Cardiol 10(2):161-167

20. Sood A, Singh D, Dutta U, Mittal BR, Parmar M, Kaur G et al (2019) Effect of ursodeoxycholic acid in facilitating early hepatic clearance of radiotracer among patients undergoing $99 \mathrm{~m}$ Tc-sestamibi myocardial perfusion scintigraphy: a randomized double blind placebo controlled parallel trial. J Nucl Cardiol:1-12

21. Purbhoo K, Di Tamba M, Vangu W (2015) Efficacy of full-fat milk and diluted lemon juice in reducing infra-cardiac activity of $99 \mathrm{mtc}$ sestamibi during myocardial perfusion imaging. Cardiovasc J Afr 26(4):171

22. Fard BT, Sabet KA, Ay M (2019) Introducing a dedicated cardiac SPECT imaging system: ProSPECT. Front Biomed Technol 6(3):156-160

23. Zohaib A, Saeed MH, Shahid SA, Shahbaz M, Ehsan F, labal M (2016) Myocardial perfusion imaging (MPI); superior clinical performance, a comparison between filtered backprojection (FBP) and astonish iterative reconstruction method with cardiac catheterization. Prof Med J 23(4)

\section{Publisher's Note}

Springer Nature remains neutral with regard to jurisdictional claims in published maps and institutional affiliations.

\section{Submit your manuscript to a SpringerOpen ${ }^{\circ}$ journal and benefit from:}

- Convenient online submission

- Rigorous peer review

- Open access: articles freely available online

- High visibility within the field

- Retaining the copyright to your article

Submit your next manuscript at $\boldsymbol{\nabla}$ springeropen.com 